\title{
gु
}

\section{Proposed Parametric Cooling of Bilayer Cuprate Superconductors by Terahertz Excitation}

\author{
S. J. Denny, ${ }^{1, *}$ S. R. Clark, ${ }^{1,2}$ Y. Laplace, ${ }^{2}$ A. Cavalleri, ${ }^{2,1}$ and D. Jaksch ${ }^{1,3}$ \\ ${ }^{1}$ Clarendon Laboratory, University of Oxford, Parks Road, Oxford OX1 3PU, United Kingdom \\ ${ }^{2}$ Max-Planck Institute for the Structure and Dynamics of Matter, 22761 Hamburg, Germany \\ ${ }^{3}$ Centre for Quantum Technologies, National University of Singapore, 3 Science Drive 2, Singapore 117543, Singapore
}

(Received 19 November 2014; published 31 March 2015)

\begin{abstract}
We propose and analyze a scheme for parametrically cooling bilayer cuprates based on the selective driving of a $c$-axis vibrational mode. The scheme exploits the vibration as a transducer making the Josephson plasma frequencies time dependent. We show how modulation at the difference frequency between the intrabilayer and interbilayer plasmon substantially suppresses interbilayer phase fluctuations, responsible for switching $c$-axis transport from a superconducting to a resistive state. Our calculations indicate that this may provide a viable mechanism for stabilizing nonequilibrium superconductivity even above $T_{c}$, provided a finite pair density survives between the bilayers out of equilibrium.
\end{abstract}

DOI: 10.1103/PhysRevLett.114.137001

PACS numbers: 74.25.N-, 74.50.+r, 74.72.-h, 74.81.Fa

Introduction.-The ability to use light to drive with precision a single low-energy degree of freedom of a solid is rapidly becoming an important tool for both basic research and potential technological applications [1-7]. Much work in this area has been dedicated to the excitation of lattice vibrations, which deform the crystal lattice when driven to large amplitudes [8]. Often, these vibrational modes lie in the midinfrared region. The laser excitation of these modes can be coherent and highly selective and induce little direct heating, in contrast to near-visible wavelengths [9-13]. Accordingly, such nonlinear phononic techniques have been directed toward materials with strong electronic correlations, with the goal of optically switching their collective properties, including superconductivity, ferroelectricity, or colossal magnetoresistance $[5,6]$.

Notably, the application of nonlinear lattice control in high- $T_{c}$ superconducting cuprates has led to the realization of light enhanced superconductivity, demonstrated first by targeting modes which dynamically "unbuckle" the crystallographic structure of the cuprate $\mathrm{La}_{1.675} \mathrm{Eu}_{0.2} \mathrm{Sr}_{0.125} \mathrm{CuO}_{4}$, tipping the system from striped to superconducting behavior [3,5]. In a recent experiment, coherent excitation of apical oxygen distortions in the bilayer cuprate $\mathrm{YBa}_{2} \mathrm{Cu}_{3} \mathrm{O}_{6+d}$ (YBCO) was shown to induce a transient phase which exhibited superconducting fluctuations at temperatures up to $300 \mathrm{~K}[6,7]$. In these experiments, the disruption of a competing order cannot fully explain the extraordinary temperature scale of the effect, and other phenomena related to the nature of the dynamically driven state should be considered. Consequently, here we explore the properties of bilayer cuprates under periodic driving, which in other materials systems has been shown to give rise to a renormalized electronic structure [14].

While the physics of high- $T_{c}$ superconductors is not fully understood, it is generally considered that their properties are determined by the doped copper-oxide planes [15-19]. As these planes are weakly coupled through insulating layers in the $c$ axis, the low-energy $c$-axis electrodynamics of cuprates can often be adequately described as a stack of intrinsic Josephson junctionsmaking them potentially compact sources of coherent continuous-wave $\mathrm{THz}$ frequency radiation [20-22]. Owing to the relatively small $c$-axis phase stiffness and poor screening phase fluctuations in the copper oxide planes are likely to play a significant role in determining the critical temperature $T_{c}$ [23,24]. In this Letter, we propose a cooling scheme for bilayer cuprates, similar to the laser cooling of solids via anti-Stokes fluorescence [25], but specifically targeting the crucial order-parameter phase.

Cooling of phase fluctuations by driving has been demonstrated in a BCS superconductor using microwave frequency sideband techniques, leading to an increase in the critical current $I_{c}$ for a single Josephson junction [26]. Bilayer cuprates shown schematically in Fig. 1(a) are composed of unit cells with two junctions whose insulators alternate between thick interbilayers and thin intrabilayers. Typically, they have low $(l)$ and high $(h)$ Josephson plasma normal modes in the region $\omega_{l} \approx 2 \pi \times 1 \mathrm{THz}$ and $\omega_{h} \approx 2 \pi \times 10 \mathrm{THz}$, respectively. Our key idea is to use the selective driving of a $c$-axis vibrational mode as a transducer to modulate plasma frequencies in time. Although this driving differs from that used to laser cool many-body systems of atoms [27-29], ions [30], and optomechanical oscillators [31], the resulting effect is analogous. Given a temperature $\hbar \omega_{l}<k_{B} T<\hbar \omega_{h}$, parametric modulation [32] of the bilayer structure can cool by up-converting energy from the thermally populated interbilayer plasmon modes-responsible for phase fluctuations - to the intrabilayer plasmon modes [see Fig. 1(b)]. We make testable quantitative predictions on the efficiency of 
(a)

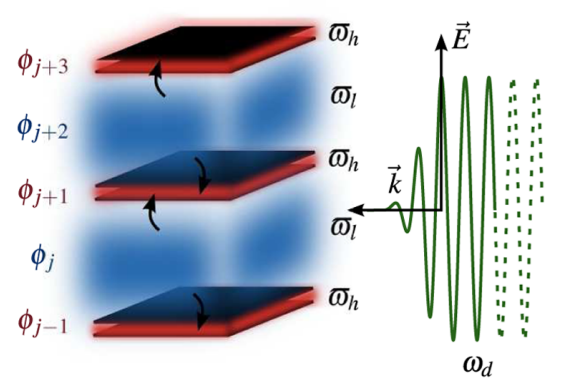

(b)

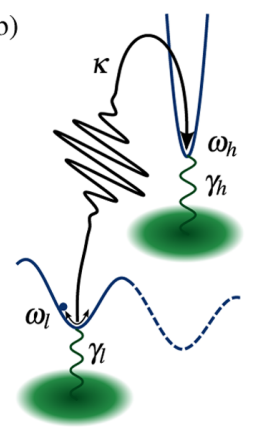

FIG. 1 (color online). (a) A schematic of a bilayer cuprate such as YBCO composed of Josephson junctions, each with a phase difference $\phi_{j}$ and alternating interbilayer (low) $\varpi_{l}$ and intrabilayer (high) $\varpi_{h}$ plasma frequencies. (b) Parametric cooling scheme where the coupling between the low and high frequency normal modes $\omega_{l}$ and $\omega_{h}$ is modulated. Tuning the modulation to $\omega_{d}=\frac{1}{2}\left(\omega_{h}-\omega_{l}\right) \mathrm{THz}$ cools the low frequency mode by up-converting fluctuations to the high frequency one.

cooling and the resulting elevations in $I_{c}$. Importantly, we find that optimal suppression of phase fluctuations occurs for modulation at the frequency difference of the plasmon modes. While the theory discussed here is inspired by the experiments reported in Refs. [6,7], optimal conditions were not met in these experiments so the theory outlined may or may not explain those observations.

Model. - The $c$-axis electrodynamics of cuprate materials are commonly modeled as alternating stacks of superconducting and insulating layers, with the Josephson effect and quasiparticle tunneling providing coupling along the $c$ axis [20,33]. The application of Maxwell's equations, augmented by the Josephson relations, yields a model in which there is both inductive and capacitive coupling between the phases of each intrinsic junction [34]. To simplify our treatment, we consider a sufficiently small crystal in the $a$ and $b$ dimensions $(<100 \mu \mathrm{m}$ for many cuprates) such that plasmon modes with finite quasimomentum in the $a b$ plane are energetically prohibited. Consequently, the spatial dependence of the phases can be neglected, reducing the system to a stack of short junctions, similar to those fabricated in heterostructures, with layer charging providing the dominant coupling [34].

In addition to the Josephson dynamics, there are also phonon modes spanning the $\mathrm{THz}$ range [35-38], many of which describe $c$-axis vibrations within the insulating layers [39]. The direct coupling of an infrared active vibrational coordinate $q$ to the Josephson plasma frequencies is central to the physics of this work. Such a coupling, which will be $q^{2}$ due to symmetry, might arise in numerous ways. For example, the motion of apical oxygens may modulate superfluid density and the plasma frequency. Alternatively, lattice vibrations may be modeled as a time-dependent modulation of the capacitance of the insulating layers, equivalent to a $\chi^{(3)}$ optical nonlinearity [40]. Either type of modulation results in the same general

effect, and for concreteness, we focus on the latter mechanism [41]. This is equivalent to the insulating layer $I$ having an effective time-dependent relative permittivity $\epsilon_{I}(t)$ modulated at twice the driving frequency $\omega_{d}$, along with off-resonant harmonics which we neglect. In Ref. [41], we show that the Josephson phase dynamics is described by a modified Koyama-Tachiki model [34],

$$
\begin{aligned}
\partial_{t}^{2} \phi_{I}= & -\alpha \varpi_{I-1}^{2} \sin \left(\phi_{I-1}\right)+\left[2 \alpha+1+\eta_{I}(t)\right] \varpi_{I}^{2} \sin \left(\phi_{I}\right) \\
& -\alpha \varpi_{I+1}^{2} \sin \left(\phi_{I+1}\right),
\end{aligned}
$$

where $\phi_{I}$ is the gauge-invariant phase difference across the Ith insulating layer and $\varpi_{I}=c / \sqrt{\epsilon_{I}} \lambda_{c}$ is the layer's alternating plasma frequency given by the static permittivity $\epsilon_{I}$, the superconducting $c$-axis penetration depth $\lambda_{c}$, and the speed of light in vacuum $c$. The capacitive coupling between junctions is quantified by the parameter $\alpha$, which takes values in the range $0.1-5$ for common high- $T_{\mathrm{c}}$ superconductors $[43,44]$. The relative driving strength is $\eta_{I}(t)=(2 \alpha+1) \Lambda_{I}\left[\epsilon_{I} / \epsilon_{I}(t)-1\right]=\eta_{I} \sin ^{2}\left(\omega_{d} t\right)$, where the factor $\Lambda_{I}<1$ accounts for the enlarged effective thickness of a layer due to $\lambda_{c}$ [41]. The driving therefore modulates the bare plasma frequency $\varpi_{I}$. An alternative approach, making use of the Lawrence-Doniach model, yields an identical equation of motion [20,45].

Two-junction unit cell.-To examine the phase dynamics of a unit cell, we linearize Eq. (1) and move to the normal mode frame of Eq. (1), with $\eta_{I}(t)$ replaced by its time average $\frac{1}{2} \eta_{I}$. We adopt a classical Langevin framework for describing the noise $\xi_{l, h}(t)$ and damping $\gamma_{l, h}$ caused, e.g., by long-wavelength phonons and incoherent quasiparticle currents. This gives

$$
\begin{aligned}
\partial_{t}^{2} \varphi_{l}-\gamma_{l} \partial_{t} \varphi_{l}+\omega_{l}^{2}(t) \varphi_{l}+\Delta_{h}(t) \varphi_{h} & =\xi_{l}(t), \\
\partial_{t}^{2} \varphi_{h}-\gamma_{h} \partial_{t} \varphi_{h}+\omega_{h}^{2}(t) \varphi_{h}+\Delta_{l}(t) \varphi_{l} & =\xi_{h}(t),
\end{aligned}
$$

where $\varphi_{l, h}$ are the normal mode phase coordinates. Importantly, the driving introduces a time-dependent off-diagonal coupling $\Delta_{l, h}(t)=\Delta_{l, h} \cos \left(2 \omega_{d} t\right)$, where $\Delta_{l}=-\frac{1}{2} \Theta(\alpha) \eta_{h} \varpi_{l}^{2}$, using $\Theta(\alpha)=\alpha^{2} /(2 \alpha+1)^{2}$, and $\Delta_{h}=-\frac{1}{2} \eta_{h} \varpi_{h}^{2}$ to lowest order in $r=\varpi_{l} / \varpi_{h}$. Similarly, the driving also induces a modulation of the normal mode frequencies $\omega_{l, h}^{2}(t)=\omega_{l, h}^{2}-\frac{1}{2} A_{l, h}^{2} \cos \left(2 \omega_{d} t\right)$, with $A_{l}^{2}=$ $\left[\eta_{l}+\Theta(\alpha) \eta_{h}\right] \varpi_{l}^{2}$ and $A_{h}^{2}=\eta_{h} \varpi_{h}^{2}$. The quadratic nature of the driving shifts the normal mode frequencies $\omega_{l, h}$, in line with experimental observations [6,7], as explicitly shown in Ref. [41]. The noise $\xi_{l, h}(t)$ is approximated as independent, white, and Gaussian, $\left\langle\xi_{l, h}(t) \xi_{l, h}\left(t^{\prime}\right)\right\rangle=$ $\Gamma_{l, h} \delta\left(t-t^{\prime}\right)$, and is related to the damping by the fluctuation-dissipation theorem as $\Gamma_{l}=2 \gamma_{l} \omega_{l}^{2}\left(T / T_{0}\right)$ and $\Gamma_{h}=2 \gamma_{h} \omega_{l}^{2}\left(\omega_{l} / \omega_{h}\right)^{2}\left(T / T_{0}\right)$, where $T_{0}$ is a systemdependent temperature scale set by the capacitive energy associated with the mode $\varphi_{l}$. In the absence of driving, 


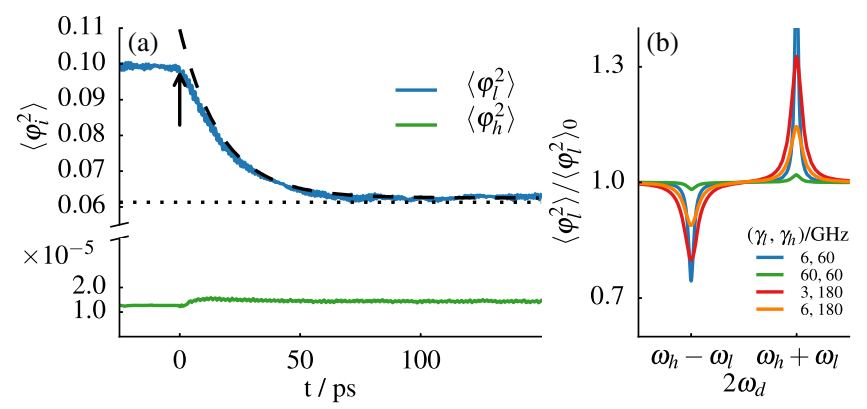

FIG. 2 (color online). Parametric cooling in a two-junction unit cell with (undriven) normal modes $\omega_{l}=2 \pi \times 1 \mathrm{THz}$, $\omega_{h}=2 \pi \times 10 \mathrm{THz}, \quad \gamma_{l}=0.19 \mathrm{THz}, \quad \gamma_{h}=3.63 \mathrm{THz}, \quad \alpha=1$, $\eta_{l}=0$, and $\eta_{h}=0.1$. (a) Phase and conjugate momentum quadratures of the interbilayer and intrabilayer modes. Before $t=0$ (indicated), $T_{\text {initial }} / T_{0}=0.1$; then parametric driving is applied, after which fluctuations on both quadratures of the interbilayer (intrabilayer) mode are cooled to $0.6 T_{\text {initial }}$ (heated to $\left.1.2 T_{\text {initial }}\right)$. Analytic predictions of the steady state (dotted line) and asymptotic cooling rate (dashed line) are also shown. (b) Cooling or heating sidebands of the interbilayer mode as the driving frequency $\omega_{d}$ is varied.

the damping and noise will thermalize the system at a temperature $T$. In contrast to well-isolated quantum optical or atomic systems, their continued presence during driving accounts for the persistent reheating expected in a solid-state system.

We integrate the stochastic differential equations Eq. (2) with a quasisymplectic velocity Verlet propagator [46]. In Fig. 2, we report results for a representative set of relevant parameters for bilayer cuprates when driving at the difference frequency $\omega_{d}=\frac{1}{2}\left(\omega_{h}-\omega_{l}\right)$. Since the intrabilayer junction is typically more highly damped, we have taken $\gamma_{h}>\gamma_{l}$. As shown in Fig. 2(a), once the driving is switched on, the phase fluctuations of the interbilayer mode $\varphi_{l}$ are strongly suppressed. Up-conversion correspondingly causes fluctuations to increase on the intrabilayer mode $\varphi_{h}$; however, its fluctuations remain small even in the driven steady state. Number fluctuations (not shown) for the two modes behave similarly. Although the resulting steady state is nonthermal, the level of fluctuations is consistent with $\varphi_{l}$ being substantially cooled and $\varphi_{h}$ being heated. The $\omega_{d}$ dependence of the effect is shown in Fig. 2(b), where cooling (red) and heating (blue) sidebands are observed at $\omega_{d}=\frac{1}{2}\left(\omega_{h}-\omega_{l}\right)$ and $\omega_{d}=\frac{1}{2}\left(\omega_{h}+\omega_{l}\right)$, respectively.

Parametric cooling.-We estimate the final temperature and cooling rate by neglecting the modulation of the normal mode frequencies $\omega_{m}(t)$ and retaining only the modulation of the couplings $\Delta_{m}(t)$. The resulting model is an effective coupled-oscillator Hamiltonian, as depicted in Fig. 1(b),

$$
\begin{aligned}
H= & \frac{p_{l}^{2}}{2 \Delta_{l}}+\frac{p_{h}^{2}}{2 \Delta_{h}}+\frac{1}{2} \Delta_{l} \omega_{l}^{2} \varphi_{l}^{2}+\frac{1}{2} \Delta_{h} \omega_{h}^{2} \varphi_{h}^{2} \\
& +\cos \left(2 \omega_{d} t\right) \Delta_{l} \Delta_{h} \varphi_{l} \varphi_{h},
\end{aligned}
$$

where $p_{l, h}$ is the conjugate momentum to $\varphi_{l, h}$. Applying the rotating wave approximation and transforming to the frame rotating with the coupling reveals that $\omega_{d}=\frac{1}{2}\left(\omega_{h}-\omega_{l}\right)$ modulation induces resonant exchange energy between the oscillators [41]. Since both oscillators are coupled to the same thermal reservoir, up-conversion of energy from $\varphi_{l}$ to $\varphi_{h}$ is the dominant process. At the temperatures of interest, the high frequency bath modes thermalizing $\varphi_{h}$ are effectively unoccupied, so excess up-converted energy is dissipated. This leads to cooling controlled by the normalized coupling $\kappa_{0}^{2}=\left(\Delta_{h} \Delta_{l}\right) /\left(\omega_{l} \omega_{h}\right)=\frac{1}{4}\left(\eta_{h}^{2} \omega_{h}^{2}\right) g(\alpha) r+\mathcal{O}\left(r^{3}\right)$, with $g(\alpha)=\Theta(\alpha) / \sqrt{3 \alpha^{2}+4 \alpha+1}$, and is maximized at $\alpha=\alpha_{0} \approx 1.07$, where $g\left(\alpha_{0}\right) \approx 0.04$. Note that $\kappa_{0}$ depends only on $\eta_{h}$ to leading order in $r$ because the $\varphi_{h}$ mode is more massive by a ratio $\left(\omega_{h} / \omega_{l}\right)^{2}$, so modulation of the intrabilayer insulator is predicted to be most effective. In the Ref. [41], we show that the asymptotic cooling rate is $\gamma_{\mathrm{dr}}=\gamma_{l}+\kappa_{0}^{2} /\left(\gamma_{h}-\gamma_{l}\right)+\mathcal{O}\left(\kappa_{0}^{3}\right)$, and the steady-state fluctuations for the interbilayer mode are [32]

$$
\left\langle\varphi_{l}^{2}\right\rangle /\left\langle\varphi_{l}^{2}\right\rangle_{0}=1-\mathcal{S}\left(1-\omega_{l} / \omega_{h}\right),
$$

where the scale factor $0 \leq \mathcal{S} \leq 1$ is given by $\mathcal{S}=\zeta / \gamma_{1} \chi$, with $\zeta=\kappa_{0}^{2}\left(\gamma_{l}+\gamma_{h}\right) /\left[\Delta \omega^{2}+\left(\gamma_{l}+\gamma_{h}\right)^{2}\right], \chi=1+\zeta\left(\gamma_{l}+\gamma_{h}\right) /$ $\gamma_{l} \gamma_{h}$, and $\Delta \omega=2 \omega_{d}-\left(\omega_{h}-\omega_{l}\right)$. Note that $\mathcal{S}=0$, indicating no cooling, if either $\gamma_{h}=0$ or $\kappa_{0}=0$, while for an undamped interbilayer mode $\gamma_{l}=0$ with $\gamma_{h}, \kappa_{0}>0$, we have $\mathcal{S}=1$ giving the maximum suppression of fluctuations. With increasing $\gamma_{l}>0, \mathcal{S}$ decreases monotonically, implying that the interbilayer should be underdamped, and $\mathcal{S}$ displays the expected resonance around $\Delta \omega=0$. The predictions of this analysis are included in Fig. 2(a), and they agree with the numerical solution to within a few percent over a wide parameter regime. Moreover, in Ref. [41], we show that the neglect of quantum fluctuations in either plasmon mode does not affect the validity of our approach.

Cooling a stack of junctions.-We now turn to the main result of this Letter and consider the full nonlinear dynamics of a stack of 100 junctions described by Eq. (1) using the classical Langevin treatment outlined. As shown in Fig. 3(a) (inset) the linearized normal modes of the stack now form bands, $\varphi_{l, i}$ and $\varphi_{h, i}$, of low and high frequency plasmons which we take as being uniformly damped at rates $\gamma_{l}$ and $\gamma_{h}$, respectively. The $c$-axis critical temperature $T_{c}$ of the stack was determined by locating the point where spontaneous thermal phase slipping first induces a resistive state under negligible bias [41]. In Fig. 3(a), we show the fluctuations of the modes $\varphi_{l, i}$ for the case where the driving $\omega_{d}$ is tuned to half the difference frequency near the lower edge of the bands (indicated). As with the two-junction case a suppression of phase fluctuations is observed for nearby modes. The steady-state driven fluctuations of $\varphi_{l, i}$ when $\omega_{d}$ targets different regions of the interbilayer band are shown in Fig. 3(b). 

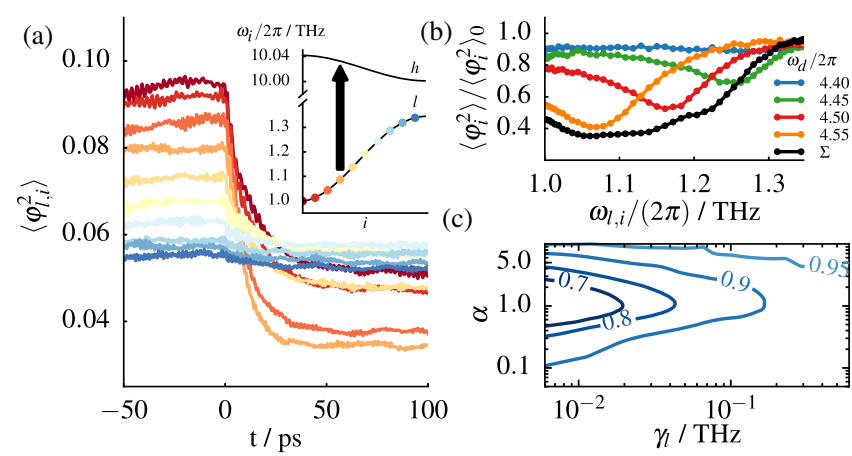

FIG. 3 (color online). (a) Reduction of $\varphi_{l, i}$ fluctuations for a 100 -junction stack over time, at $T / T_{c} \approx 0.7$. A selection of 10 modes have been displayed across the interbilayer band as shown in the inset. (b) In the steady-state for relative fluctuations for each mode in the interbilayer band is plotted for a selection of $\omega_{d}$. (c) A contour plot of the $\left\langle\sin ^{2} \varphi_{l, i}\right\rangle /\left\langle\sin ^{2} \varphi_{l, i}\right\rangle_{0}$ averaged across the interbilayer band sweeping over the damping $\gamma_{l}$ and capacitive coupling $\alpha$. Here we have taken $\gamma_{h}=0.1 \min \left(\omega_{h, i}\right)$, $\min \left(\omega_{h, i}\right) / \min \left(\omega_{l, i}=10\right.$. For each value of $\alpha$, we drive at a frequency targeting the bottom of the $l$ band.

Also plotted is the response for colored driving equally superposing three different values of $\omega_{d}$, showing that broadband driving can induce suppression over a wide range of the band. The systematic variation of the suppression with $\alpha$ and $\gamma_{l}$ is shown in Fig. 3(c), where the relative phase fluctuations averaged over all modes in the interbilayer band are plotted. This indicates that optimal cooling occurs with a moderate coupling and weak intrinsic damping of the interbilayer plasmon.

A complementary characterization of the stack is given by the $c$-axis superconducting transport properties quantified by the switching current distribution. This was obtained by sweeping in a time $\Delta t=1 \mathrm{~ns}$ the bias current $I(t)$ linearly in time from zero up to the critical current $I_{c}$. The tilt of the washboard potential of every junction in the stack increases until a phase slip event occurs, at which point the potential difference across the stack becomes finite. In Fig. 4(a), the Q-Q plot for the computed statistics of this process is shown. This compares the quantiles of the original thermal distribution to those of the stack subjected to different driving strengths $\eta_{h}$. The curves indicate a shift in the mean of the switching distribution and a reduction in its spread, both of which are expected for a switching distribution at a lower temperature (see Ref. [47], pp. 207-209).

This tendency is confirmed in Fig. 4(b), where the cumulative distribution function (CDF) for the switching current is plotted for the same set of drivings. The distribution is shifted to higher values of the bias current with increasing driving, analogous to the shift that is observed for thermal curves with decreasing temperature. The suppression of phase fluctuations in the interbilayer band thus corresponds to a measurable cooling effect on an experimentally relevant figure of merit. Since $\hbar \omega_{h, i}>k_{B} T$,
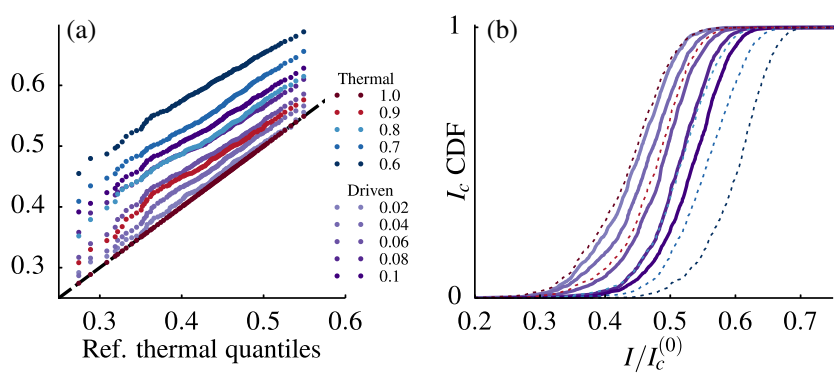

FIG. 4 (color online). (a) Q-Q plot of switching current distributions for driving strengths $\eta_{h}=0 \rightarrow 0.1$, at $T / T_{c} \approx 0.7$. Thermal curves are relative to this temperature. The dashed line indicates the initial thermal distribution. (b) Numerically computed CDF of switching current at $\alpha=1$ with a sweep time $\Delta t=1 \mathrm{~ns}$. Solid lines are the driven stack with different driving strengths as in (a), while the dotted lines correspond to thermal benchmarks.

the intrabilayer modes remain superconducting with no phase slips induced.

Conclusion.-We have shown the suppression of phase fluctuations of Josephson plasmons in bilayer cuprates by selectively targeting IR-active $c$-axis vibrational modes that modulate the Josephson plasma frequencies. At the difference frequency between intrabilayer and interbilayer plasma modes, this driving can be exploited to implement parametric cooling. We have shown that a moderate capacitive coupling and low damping of the interbilayer modes is needed for this effect to be optimal. While the coupling in BSCCO-2212 is too weak, both YBCO and TBCCO-2201 satisfy these requirements, making them strong candidate materials. Additionally, they both possess phonon modes near the difference frequency with atomic motion in the intrabilayer junction. Related effects have already been observed in YBCO [48], and the experiments reported in Refs. [6,7] may in fact rest on a related physical mechanism, even though the exact resonance condition for parametric cooling is not strictly met.

The proposed scheme may provide a pathway for dynamically stabilizing superconductivity above $T_{c}$ so long as superconducting coherence and a high frequency plasmon remain [49]. For cooling to stay effective, the interbilayer plasmon must remain underdamped even with increasing temperature. Future work includes extending the treatment to long junctions possessing phase fluctuations in the $a b$ plane [50] and investigating the possibility of darkstate cooling schemes [28] in the richer structure of trilayer materials.

This research is funded by the European Research Council under the European Union's Seventh Framework Programme (FP7/2007-2013)/ERC Grant Agreement No. 319286 Q-MAC, and resources were provided by the TNT project funded via EPSRC Projects No. EP/ K038311/1 and No. EP/J010529/1. 
*s.denny@physics.ox.ac.uk

[1] A. Subedi, A. Cavalleri, and A. Georges, Phys. Rev. B 89, 220301 (2014).

[2] M. Rini, R. Tobey, N. Dean, J. Itatani, Y. Tomioka, Y. Tokura, R. W. Schoenlein, and A. Cavalleri, Nature (London) 449, 72 (2007).

[3] M. Först, R. I. Tobey, S. Wall, H. Bromberger, V. Khanna, A. L. Cavalieri, Y.-D. Chuang, W. S. Lee, R. Moore, W. F. Schlotter, J. J. Turner, O. Krupin, M. Trigo, H. Zheng, J. F. Mitchell, S. S. Dhesi, J. P. Hill, and A. Cavalleri, Phys. Rev. B 84, 241104 (2011).

[4] A. D. Caviglia, R. Scherwitzl, P. Popovich, W. Hu, H. Bromberger, R. Singla, M. Mitrano, M. C. Hoffmann, S. Kaiser, P. Zubko, S. Gariglio, J.-M. Triscone, M. Först, and A. Cavalleri, Phys. Rev. Lett. 108, 136801 (2012).

[5] D. Fausti, R. I. Tobey, N. Dean, S. Kaiser, A. Dienst, M. C. Hoffmann, S. Pyon, T. Takayama, H. Takagi, and A. Cavalleri, Science 331, 189 (2011).

[6] S. Kaiser, C. R. Hunt, D. Nicoletti, W. Hu, I. Gierz, H. Y. Liu, M. Le Tacon, T. Loew, D. Haug, B. Keimer, and A. Cavalleri, Phys. Rev. B 89, 184516 (2014).

[7] W. Hu, S. Kaiser, D. Nicoletti, C. R. Hunt, I. Gierz, M. C. Hoffmann, M. Le Tacon, T. Loew, B. Keimer, and A. Cavalleri, Nat. Mater. 13, 705 (2014).

[8] M. Forst, C. Manzoni, S. Kaiser, Y. Tomioka, Y. Tokura, R. Merlin, and A. Cavalleri, Nat. Phys. 7, 854 (2011).

[9] K. Miyano, T. Tanaka, Y. Tomioka, and Y. Tokura, Phys. Rev. Lett. 78, 4257 (1997).

[10] A. Cavalleri, C. Tóth, C. W. Siders, J. A. Squier, F. Ráksi, P. Forget, and J. C. Kieffer, Phys. Rev. Lett. 87, 237401 (2001).

[11] S. Iwai, S. Tanaka, K. Fujinuma, H. Kishida, H. Okamoto, and Y. Tokura, Phys. Rev. Lett. 88, 057402 (2002).

[12] M. Chollet, L. Guerin, N. Uchida, S. Fukaya, H. Shimoda, T. Ishikawa, K. Matsuda, T. Hasegawa, A. Ota, H. Yamochi, G. Saito, R. Tazaki, S.-I. Adachi, and S.-Y. Koshihara, Science 307, 86 (2005).

[13] L. Perfetti, P. A. Loukakos, M. Lisowski, U. Bovensiepen, H. Berger, S. Biermann, P. S. Cornaglia, A. Georges, and M. Wolf, Phys. Rev. Lett. 97, 067402 (2006).

[14] Y. H. Wang, H. Steinberg, P. Jarillo-Herrero, and N. Gedik, Science 342, 453 (2013).

[15] E. Pavarini, I. Dasgupta, T. Saha-Dasgupta, O. Jepsen, and O. K. Andersen, Phys. Rev. Lett. 87, 047003 (2001).

[16] C. Weber, K. Haule, and G. Kotliar, Phys. Rev. B 82, 125107 (2010).

[17] J. A. Slezak, J. Lee, M. Wang, K. McElroy, K. Fujita, B. M. Andersen, P. J. Hirschfeld, H. Eisaki, S. Uchida, and J. C. Davis, Proc. Natl. Acad. Sci., USA 105, 3203 (2008).

[18] M. Mori, G. Khaliullin, T. Tohyama, and S. Maekawa, Phys. Rev. Lett. 101, 247003 (2008).

[19] H. Sakakibara, H. Usui, K. Kuroki, R. Arita, and H. Aoki, Phys. Rev. Lett. 105, 057003 (2010).

[20] X. Hu and S.-Z. Lin, Supercond. Sci. Technol. 23, 053001 (2010).

[21] U. Welp, K. Kadowaki, and R. Kleiner, Nat. Photonics 7, 702 (2013).

[22] L. N. Bulaevskii and A. E. Koshelev, Phys. Rev. Lett. 99, 057002 (2007).

[23] V. J. Emery and S. A. Kivelson, Nature (London) 374, 434 (1995).
[24] A. Mihlin and A. Auerbach, Phys. Rev. B 80, 134521 (2009).

[25] G. Nemova and R. Kashyap, Rep. Prog. Phys. 73, 086501 (2010).

[26] J. Hammer, M. Aprili, and I. Petković, Phys. Rev. Lett. 107, 017001 (2011).

[27] C. E. Wieman, D. E. Pritchard, and D. J. Wineland, Rev. Mod. Phys. 71, S253 (1999).

[28] A. Griessner, A. J. Daley, S. R. Clark, D. Jaksch, and P. Zoller, Phys. Rev. Lett. 97, 220403 (2006).

[29] D. Jaksch, S. A. Gardiner, K. Schulze, J. I. Cirac, and P. Zoller, Phys. Rev. Lett. 86, 4733 (2001).

[30] J. Eschner, G. Morigi, F. Schmidt-Kaler, and R. Blatt, J. Opt. Soc. Am. B 20, 1003 (2003).

[31] M. Aspelmeyer, T. J. Kippenberg, and F. Marquardt, Rev. Mod. Phys. 86, 1391 (2014).

[32] S. P. Vyatchanin, Dokl. Akad. Nauk. SSSR 234, 1295 (1977) [Sov. Phys. Dokl. 22, 321 (1977)].

[33] R. Kleiner and P. Müller, Phys. Rev. B 49, 1327 (1994).

[34] T. Koyama and M. Tachiki, Phys. Rev. B 54, 16183 (1996).

[35] W. Kress, U. Schröder, J. Prade, A. D. Kulkarni, and F. W. de Wette, Phys. Rev. B 38, 2906 (1988).

[36] J. Prade, A. D. Kulkarni, F. W. de Wette, U. Schröder, and W. Kress, Phys. Rev. B 39, 2771 (1989).

[37] A. D. Kulkarni, J. Prade, F. W. de Wette, W. Kress, and U. Schröder, Phys. Rev. B 40, 2642 (1989).

[38] A. D. Kulkarni, F. W. de Wette, J. Prade, U. Schröder, and W. Kress, Phys. Rev. B 43, 5451 (1991).

[39] R. Liu, C. Thomsen, W. Kress, M. Cardona, B. Gegenheimer, F. W. de Wette, J. Prade, A. D. Kulkarni, and U. Schröder, Phys. Rev. B 37, 7971 (1988).

[40] R. W. Boyd, Nonlinear Optics (Academic Press, San Diego, 2003).

[41] See Supplemental Material http://link.aps.org/supplemental/ 10.1103/PhysRevLett.114.137001 for a more detailed discussion of the results presented in this Letter, which includes Refs. [32,34,42].

[42] M. Machida and S. Sakai, Phys. Rev. B 70, 144520 (2004).

[43] A. A. Tsvetkov, D. van der Marel, K. A. Moler, J. R. Kirtley, J. L. de Boer, A. Meetsma, Z. F. Ren, N. Koleshnikov, D. Dulic, A. Damascelli, M. Gruninger, J. Schutzmann, J. W. van der Eb, H. S. Somal, and J. H. Wang, Nature (London) 395, 360 (1998).

[44] M. Machida and T. Koyama, Phys. Rev. B 70, 024523 (2004).

[45] L. N. Bulaevskii, M. Zamora, D. Baeriswyl, H. Beck, and J. R. Clem, Phys. Rev. B 50, 12831 (1994).

[46] S. Melchionna, J. Chem. Phys. 127, 044108 (2007).

[47] M. Tinkham, Introduction to Superconductivity, 2nd ed. (Dover Publications, Mineola, 1996).

[48] R. Mankowsky, A. Subedi, M. Först, S. O. Mariager, M. Chollet, H. Lemke, J. Robinson, J. Glownia, M. Minitti, A. Frano, M. Fechner, N. A. Spaldin, T. Loew, B. Keimer, A. Georges, and A. Cavalleri, Nature (London) 516, 71 (2014).

[49] A. Dubroka, M. Rössle, K. W. Kim, V. K. Malik, D. Munzar, D. N. Basov, A. A. Schafgans, S. J. Moon, C. T. Lin, D. Haug, V. Hinkov, B. Keimer, T. Wolf, J. G. Storey, J. L. Tallon, and C. Bernhard, Phys. Rev. Lett. 106, 047006 (2011).

[50] R. Höppner, B. Zhu, T. Rexin, A. Cavalleri, and L. Mathey, arXiv:1406.3609. 